\title{
Quantum dynamics of a nano-rod under compression
}

\author{
Geoffrey M. Beck \\ School of Chemistry and Physics, University of KwaZulu-Natal, Pietermaritzburg \\ Campus, Private Bag X01 Scottsville, 3209 Pietermaritzburg, South Africa, E-mail: \\ geoff.m.beck@gmail.com \\ Alessandro Sergi (Corresponding author) \\ School of Chemistry and Physics, University of KwaZulu-Natal, Pietermaritzburg \\ Campus, Private Bag X01 Scottsville, 3209 Pietermaritzburg, South Africa, E-mail: \\ sergi@ukzn.ac.za, Tel: +2733260 5875, Fax: +27332605876
}

\begin{abstract}
A nano-rod under compression, which is capacitively coupled to a Cooperpair box, can be modeled in terms of a quartic oscillator linearly interacting with a tunnelling pseudo-spin. We have integrated numerically the quantum dynamics of this system in the partial Wigner representation and calculated the pseudo-spin population difference. Depending on the coupling, we have verified that the quantum tunnelling of the oscillator can lead to a more effective reduction of the Rabi oscillation amplitude of the pseudo-spin. Such findings suggests an experimental set-up that might be able to discriminate between the quantum and the classical motion of a nano-rod.
\end{abstract}

Keywords: Nano-oscillator, quantum dynamics, Wigner representation

\section{Introduction}

While there has been substantial progress in cooling mechanical oscillators close to the point where quantum-mechanical zero-point effects are important [1, 2, 3, 4, 5, 6], the problem of witnessing the quantum mechanical features in an unambiguous way remains somewhat unsolved. Solution of such a problem is desirable since the observation of quantum effects in mesoscopic mechanical human-made objects could lead to testing the basic principles of quantum mechanics [7, 8, 9]. With respect to this, it has been known since the beginning of quantum mechanics that distinguishing 
between quantum and classical features of isolated harmonic oscillators is difficult. For example, the law of evolution of a quantum harmonic oscillator in the Wigner formulation of quantum mechanics [10] looks perfectly classical. Nevertheless, theoretical studies have been performed both on nonlinear oscillators [11, 12, 13, 14, 15] and harmonic oscillators [16, 17, 18, 19]. However, when a nano-rod is under longitudinal compression, the potential energy profile can vary from a harmonic form to that of a double well [20, 21]. The two minima in the wells describe the two buckled states, which can be obtained at a particular strain [22]. In such a potential energy profile, the nano-rod can move from one well minima to the other either by thermal fluctuations or by quantum tunnelling. One can expect that, for such non-linear dynamics, quantum effects might be less ambiguous to detect. For example, in order to observe the quantum motion of the nano-rod, one could couple it capacitively to a Cooper-pair box [23] (following what other authors have considered in the case of harmonic nano-oscillators [16, 17, 18]). A Cooperpair box is composed of a small superconducting island weakly linked to a superconducting reservoir [23]. The balance between the charging energy and the tunneling strength of the Cooper-pair between the island and the reservoir controls the state of the Cooper-pair box. External gates can drive the Cooper-pair box into either its ground or excited state, with definite Cooper-pair numbers, or into a linear combination of such states. Such systems are possible candidates as controllable qubits in quantum computing devices [23].

\section{Theory}

The system comprised by the non-linear oscillating nano-rod coupled to the Cooper-pair box can be modelled by a a quartic oscillator linearly coupled to a tunnelling pseudo-spin. In this Letter, we have integrated numerically the quantum dynamics of the total system and looked for signatures of the quantum evolution of the non-linear oscillator in the reduced density matrix of the pseudo-spin. To this end, we adopted a mixed Wigner representation of quantum mechanics [24] and described the quartic oscillator in phase space. In such a representation, the quantum effects on the evolution of the quartic oscillator can be suitably obtained in terms of the higher order derivatives of the partially Wigner represented density matrix (PWRDM) $\hat{W}(R, P, t)$. 
The PWRDM is defined as:

$$
\hat{W}(R, P, t)=\frac{1}{2 \pi \hbar} \int d z e^{i P \cdot z / \hbar}\left\langle R-\frac{z}{2}|\hat{\rho}| R+\frac{z}{2}\right\rangle .
$$

The transform of operators is defined analogously. In such a representation, the law of motion is written as

$$
\begin{aligned}
\frac{\partial}{\partial t} \hat{W}(R, P, t) & =-\frac{i}{\hbar}\left[\hat{H}_{W} \exp \left(\frac{i \hbar}{2} \overleftarrow{\boldsymbol{\partial}} \cdot \boldsymbol{\epsilon} \cdot \overrightarrow{\boldsymbol{\partial}}\right) \hat{W}\right. \\
& \left.-\hat{W} \exp \left(\frac{i \hbar}{2} \overleftarrow{\boldsymbol{\partial}} \cdot \boldsymbol{\epsilon} \cdot \overrightarrow{\boldsymbol{\partial}}\right) \hat{H}_{W}\right]
\end{aligned}
$$

In Eq. (2) $\boldsymbol{\epsilon}=-\boldsymbol{\epsilon}^{T}$ is the total antisymmetric symbol in phase space, so that $\overleftarrow{\boldsymbol{\partial}} \cdot \boldsymbol{\epsilon} \cdot \overrightarrow{\boldsymbol{\partial}}$ denote the Poisson bracket operator [25]. The symbol $\hat{H}_{W}$ is the partially Wigner transformed Hamiltonian, which in this Letter is defined as

$$
\hat{H}_{W}=-\Omega \hat{\sigma}_{x}-c R \hat{\sigma}_{z}+H_{C, W} .
$$

where $\hat{\sigma}_{x}$ and $\hat{\sigma}_{z}$ are Pauli spin operators.

Quantum averages in the partial Wigner representation of quantum mechanics are calculated as

$$
\langle\hat{O}(t)\rangle=\operatorname{Tr}^{\prime} \int d R d P \hat{O}_{W}(R, P) \hat{W}(R, P, t) .
$$

In the general case, Eq. (2) is not simpler to solve that the standard Von Neumann equation [26]. However, when the Hamiltonian $H_{C, W}$ has the form

$$
H_{C, W}=\frac{P^{2}}{2}+\sum_{k=1}^{n_{k}} \frac{b_{k}}{k !} R^{k}
$$

where $n_{k}$ is a fixed integer, Eq. (2) becomes

$$
\begin{aligned}
\frac{\partial}{\partial t} \hat{W}(R, P, t) & =-\frac{i}{\hbar}\left[\hat{H}_{W}(1+\mathcal{A}) \hat{W}\right. \\
& \left.-\hat{W}(1+\mathcal{A}) \hat{H}_{W}\right],
\end{aligned}
$$


where $\mathcal{A}$ is a differential operator of at most the same order $n_{k}$ as the polynomial appearing in Eq. (5). Its definition is given by

$$
\mathcal{A}=\sum_{n=1,3,5, \ldots}^{n_{k}} \frac{1}{n !}\left(\frac{i \hbar}{2}\right)^{n}(\overleftarrow{\boldsymbol{\partial}} \cdot \boldsymbol{\epsilon} \cdot \overrightarrow{\boldsymbol{\partial}})^{n}
$$

Here, we define a quartic oscillator by setting $n_{k}=4$ in Eq. (5). Quantum dynamics is obtained upon considering all the quantum corrections in Eq. (7). while the classical evolution of the oscillator (coupled to the quantum pseudospin) is obtained by considering only $n=1$ in Eq. (17). This latter is a known hybrid scheme of motion [27, 28, 29, 30] according to which the quartic oscillator evolves classically on the two adiabatic potential energy surfaces determined by the Cooper-pair box system.

\section{Model and numerical calculations}

When represented in the basis of the eigenvectors of $\Omega \hat{\sigma}_{x}$, Eq. (6) appears as a set of coupled linear partial differential equations defining an initial value problem for the PWRDM. We have studied a model defined by the Hamiltonian in Eq. (3) with Eq. (5) specialised to

$$
H_{C, W}=\frac{P^{2}}{2}+\frac{b_{2}}{2} R^{2}+\frac{b_{4}}{4} R^{4}
$$

where all quantities in the Hamiltonian appear in dimensionless form. One can obtain the model Hamiltonian in Eq. (8) following the analysis performed in [22]. According to this, $H_{C, W}$ in Eq. (8) describes the dynamics of the fundamental mode of oscillation of the nano-rod: the variable $R$ denotes the Fourier amplitude of the fundamental displacement and $P$ is the momentum conjugated to such a displacement. The dimensionless variables appearing in Eq. (8) are related to their dimensionful counterparts by:

$$
\begin{aligned}
& R=\left(\frac{M \omega_{0}}{\hbar}\right)^{1 / 2} R^{\prime} \\
& P=\left(\hbar M \omega_{0}\right)^{-1 / 2} P^{\prime},
\end{aligned}
$$

Additionally, dimensionless parameters are similarly defined:

$$
\Omega=\frac{\Omega^{\prime}}{\omega_{0}},
$$




$$
\begin{aligned}
c & =\frac{c^{\prime}}{\omega_{0} \sqrt{M \omega_{0} \hbar}}, \\
b_{2} & =\frac{1}{M \omega_{0}^{2}} b_{2}^{\prime} \\
b_{4} & =\frac{\hbar}{M^{2} \omega_{0}^{3}} b_{4}^{\prime} .
\end{aligned}
$$

Here, primed variables denote dimensionful quantities. In this notation $\omega_{0}$ and $M$ are the fundamental frequency and mass of the quartic oscillator, entering the definition of both $b_{2}^{\prime}$ and $b_{4}^{\prime}$, and are also dimensionful. The dimensionless coefficient $b_{2}$ is related to the strain $\epsilon$ by [22]

$$
b_{2}=\frac{\epsilon_{c}-\epsilon}{\epsilon_{c}}
$$

where $\epsilon_{c}$ is the critical strain, such that when $\epsilon>\epsilon_{c}$ one has a double well potential profile for the oscillator. Setting the fundamental frequency $\omega_{0}=$ $0.5 \mathrm{GHz}$ and the mass $M=10^{-21} \mathrm{~kg}$, similar to those discussed in [22] for a silicon nano-rod, the time $\left(t=\omega_{0} t^{\prime}\right)$ is given in ns and the length scale of the nano-rod is approximately $0.1 \AA$. In the following, we will refer to the dimensionless quantities. However, the use of Eqs. (9-14) allows one to easily determine the physical values.

In this study, we use two sets of values for the quantities describing the elastic properties of the nano-rod. The first set is given by $b_{2}=-1$ corresponding to $b_{2}^{\prime}=-10^{-5} \mathrm{~kg} \mathrm{~s}^{-2}$ and $b_{4}=0.5$ corresponding to $b_{4}^{\prime}=4.742 \times 10^{15}$ $\mathrm{kg} \mathrm{m} \mathrm{m}^{-2} \mathrm{~s}^{-2}$. This set of values has allowed us to observe relevant dynamical effect on a short time scale. The second set chosen to be in agreement with the prediction of the elastic theory of materials for a nano-rod with the values of frequency and mass given above and thickness of the order of the nano-meters [22]; in this case $b_{2}=-0.01$, corresponding to $b_{2}^{\prime}=-1 \times 10^{-7}$ $\mathrm{kg} \mathrm{s}^{-2}$, and $b_{4}=0.0004$, corresponding to $b_{4}^{\prime}=4.742 \times 10^{14} \mathrm{~kg} \mathrm{~m}^{-2} \mathrm{~s}^{-2}$. This second set of values has required us to extend the calculation in the time range for almost a factor of two.

For the numerical calculation we have employed the method of lines [31] by discretizing phase space using a grid of 120 points in both the $R$ and $P$ directions. The resulting system of ordinary differential equations, in the time variable, has been integrated using a Runge-Kutta Cash-Karp [32] integrator of fifth order, with an time-step of $10^{-4}$ in dimensionless units. The time scale achieved in our calculations (of the order of $10^{1}-10^{2} \mathrm{~ns}$ ) is satisfactory, in 
that the decoherence time of a suitably prepared Cooper-pair box is of the order of $500 \mathrm{~ns}$ [17]. In our numerical simulations, we have considered an initial PWRDM with the spin in its excited state and the oscillator in a coherent state. The PWRDM is then explicitly given by

$$
\begin{aligned}
\hat{W}(R, P) & =\left[\begin{array}{ll}
1 & 0 \\
0 & 0
\end{array}\right] \times \frac{1}{\pi \hbar} \exp \left[-\frac{\left(R-R_{0}\right)^{2}}{2(\Delta R)^{2}}\right] \\
& \times \exp \left[-2(\Delta R)^{2}\left(P-P_{0}\right)^{2}\right],
\end{aligned}
$$

where $\Delta R$ represents the uncertainty in the position of the oscillator. In our calculations, we have chosen the particular value of $\Delta R$ to be 0.6071 , to aid initial confinement of the oscillator to a single well of the potential profile. Additionally, the parameter $R_{0}$ has been chosen so that the coherent state is initially located in the ground state minimum of the left well of the quartic potential. The numerical simulation of Eq. (6) can be performed with or without the inclusion of the quantum corrections on the dynamics of the quartic oscillator, corresponding to the full quantum and hybrid cases respectively.

We have performed a series of calculations as a function of the coupling parameter $c$ between the pseudo-spin and the quartic oscillator $(0.1 \leq c \leq 1)$. In such a range of parameters we have examined the effect of the quantum nature of the evolution of the quartic oscillator on the pseudo-spin population difference $\left\langle\hat{\sigma}_{z}(t)\right\rangle$ and the position-probability $\operatorname{Prob}(R)$ of the oscillator itself.

For $b_{2}=-1$ and $b_{4}=0.5$, in the time interval $t \leq 10$, we observed that the inclusion of quantum dynamical effects promotes tunnelling between the wells of the potential profile. In turn, this leads to modifications, based on the width of the oscillator distribution in phase space, of the effective damping experienced by the Rabi oscillations in the population difference. These differences allow the quantum case to damp the Rabi oscillations more effectively at short times but also allow for the possibility of promoting Rabi oscillations if longer times are studied. These dual possibilities exist because the tunnelling introduced by the quantum dynamical effects allows the oscillator distribution to shift continuously between being highly symmetric (occupying both wells) and being asymmetric (largely occupying a single well). Whereas the classical oscillator distribution was seen to be largely static after short times.

The increased damping effect is clear in Fig. 1 for $t \geq 5$, showing the results obtained for $c=0.4$. The pronounced damping near $t=10$ corresponds to the highly well-symmetric phase-space configuration, in contrast 
to the asymmetry of the classical case, intimated by the instantaneous leftwell occupation probability in Fig. 2 at around $t \geq 7$. Figure. 3 shows the time-averaged position probability of the quartic oscillator in the same calculation: the asymmetry in classical results, in contrast with the symmetry of the quantum case, again demonstrates that the quantum corrections introduce additional tunnelling effects into the dynamics. Seemingly, tunnelling effects are not absent from the classical case. However, such effects are the result of non-adiabatic interactions with the pseudo-spin, which are proportional in magnitude to the strength of the coupling between systems. Many of the general characteristics of such findings are confirmed at higher couplings. The increased damping effects are still apparent in the results for $c=0.6$, as seen in Fig. 4 for $t \geq 5$. The instantaneous occupation probabilities in Fig. 5 show clear differences between the quantum and the classical cases, larger fluctuations in the quantum result demonstrates that greater tunnelling effects are still in evidence. Additionally the pronounced damping occurring for $t \geq 5$ corresponds strongly to a period of highly symmetric well occupation in the quantum case. This calculation also displays a classical well-asymmetry in Fig. 6 but of reduced magnitude when compared to that in Fig. 3. Comparison of Figs. 1 and 4, in conjunction with 2 and 5 demonstrate the importance of tunnelling effects in distinguishing quantum behaviour in the quartic oscillator through the population difference of the pseudo-spin. As the differences in the occupation probability decrease, so do the short-time qualitative differences in the behaviour of the Rabi oscillations. The instantaneous well-occupation probability plots also demonstrate a correlation between differences in the width of the phase-space distribution and differences in the damping effects on the Rabi oscillations.

For $b_{2}=-0.01$ and $b_{4}=0.0004$, values which are in agreement with the elastic theory predictions for the properties of the nano-rod, we observe dynamical effects which are similar to those obtained for the first set of values, although on a longer time scale. Figure 7 displays the results of this calculation. The variation of the population of the two-level system is somewhat smaller than that achieved with the previous set of parameters. The necessity of integrating the equations for longer times also puts the numerical algorithm under strain and we find that the numerical error increases too much beyond $t=18$. This is just a reflection of the general difficulty in the integration of long time quantum dynamics. Nevertheless, there are clear indications that the damping of the population is stronger in the case of a quantum motion of the nano-rod. 


\section{Conclusion}

This work suggests that quantum effects in the motion of nano-rods under compression can be detected indirectly. The nano-rod can be coupled to a Cooper-pair box and the tunnelling dynamics of the latter can witness the quantum or classical features of the time evolution of the first. In the case where the nano-rod properties are far from elastic theory predictions, these differences emerge rapidly and greatly illuminate the relevant dynamics. However, one notes that it is the non-linearity of the oscillations in the nano-rod system that make this comparison of quantum and classical dynamical effects possible, as this non-linearity leads to the existence of quantum dynamical corrections. Despite this, the differences between quantum and classical evolutions persisted when the properties of the nano-rod where chosen in agreement with the elastic theory of materials, even though the non-linearity parameter is greatly reduced in this case. This is because the strain on the rod can be adjusted to ensure the non-linear effects are still detectable. However, one must take into account that this requires the nanorod to be maintained around one hundredth above the critical strain and that the dimensions of the nano-rod, necessarily having a length significantly greater than its thickness, may make experimental control more difficult.

In the case where the nano-rod properties are in agreement with the predictions of elastic material theory, our calculations need to be performed for a time scale larger by a factor of two and the variation of the two-level system population is somewhat smaller. The longer time scale puts the calculation in conflict with the general problem of the integration of long time quantum dynamics. However, within the time span which is accessible in a reliable way to the algorithm that we have used, the signs that allows one to discriminate between the quantum and the classical motion of the nano-rod remain apparent.

In the future we plan to study alternative numerical schemes to extend the time span of the calculations even further as well as to study other schemes of detection based on multi-level systems.

\section{Acknowledgments}

This work is based upon research supported by the National Research Foundation of South Africa. Part of this work has been performed during a sabbatical stay of A.S. at the Department of Physics of the University of Messina in Italy. 


\section{References}

[1] I. Wilson-Rae, N. Nooshi, W. Zwerger, and T. J. Kippenberg, Phys. Rev. Lett. 99093901 (2007).

[2] F. Marquardt, J. P. Chen, A. A. Clerk, and S. M. Girvin, Phys. Rev. Lett. 99093902 (2007).

[3] T. Rocheleau, T. Ndukum, C. Macklin, J. B. Hertzberg, A. A. Clerk, and K. C. Schwab, Nature 43672 (2010).

[4] A. D. O'Connell, M. Hofheinz, M. Ansmann, R. C. Bialczak, M. Lenander, E. Lucero, M. Neeley, D. Sank, H. Wang, M. Weides, J. Wenner, John M. Martiniss, and A. N. Cleland, Nature 464697 (2010).

[5] J. D. Teufel, T. Donner, Li Dale, J. H. Harlow, M. S. Allman, K. Cicak, A. J. Sirois, J. D. Whittaker, K. W. Lehnert, and R. W. Simmons, Nature 475359 (2011).

[6] J. Chan, T. P. Alegre Mayer, A. H. Safavi-Naeni, J. T. Hill, A. Krause, S. Gröblacher, M. Aspelmeyer, O. Painter, Nature 47889 (2011).

[7] M. P. Blencowe, Phys. Rep. 395159 (2004).

[8] K. C. Schwab and M. L. Roukes, Phys. Today 58 No. 7, 36 (2005).

[9] S. L. Adler and A. Bassi, Science 325275 (2009).

[10] E. Wigner, Phys. rev. 40749 (1932).

[11] I. Katz, A. Retzker, R. Straub, and R. Lifshitz, Phys. Rev. Lett. 99 040404 (2007).

[12] I. Katz, R. Lifshitz, A. Retzker, and R. Straub, New J. Phys. 10125023 (2008).

[13] J. Claudon, A. Zazunov, F. W. J. Hekking, and O. Buisson, Phys. Rev. B 78184503 (2008).

[14] L.-Z. Guo, Z.-G. Zheng, and X.-Qi Li, EPL 9010011 (2010).

[15] S. Rips, M. Kiffner, I. Wilson-Rae, and M. J. Hartmann, New J. Phys. 14023042 (2012). 
[16] A. D. Armour, M. P. Blencowe, and K. C. Schwab, Phys. Rev. Lett. 88 148301 (2002).

[17] E. K. Irish and K. Schwab, Phys. Rev. B 68155311 (2003).

[18] R. P. Tiwari and D. Stroud, Phys. Rev. B 77214520 (2008).

[19] S. Brouard, D. Alonso, and D. Sokolovski, Phys. Rev. B 84012114 (2011).

[20] A. Chakraborty, Mol. Phys. 1071777 (2009).

[21] A. Chakraborty, Mol. Phys. 109517 (2009).

[22] S. M. Carr, W. E. Lawrence, and M. N. Wybourne, Phys. Rev. B 64 220101(R) (2001).

[23] Y. Makhlin, G. Schön, and A. Shnirman, Rev. Mod. Phys. 73357 (2001).

[24] A. Sergi, I. Sinayskiy, and F. Petruccione, Phys. Rev. A 80012108 (2009).

[25] A. Sergi, Phys. Rev. E 72066125 (2005).

[26] H.-P. Breuer and F.Petruccione, The Theory of Open Quantum Systems (Oxford University Press, Oxford, 2002).

[27] C. C. Martens and and J.-Y. Fang, J. Chem. Phys. 1064918 (1996).

[28] R. Kapral and G. Ciccotti, J. Chem. Phys. 1108919 (1999).

[29] O.V. Prezhdo and V. V. Kisil, Phys. Rev. A 56162 (1997).

[30] I. Horenko, C. Salzmann, B. Schmidt, and C. Schutte, J. Chem. Phys. 11711075 (2002).

[31] W. E. Schiesser, The Numerical Method of Lines (Academic Press, 1991).

[32] J. R. Cash and A. H. Karp, ACM Transactions on Mathematical Software 16201 (1990). 


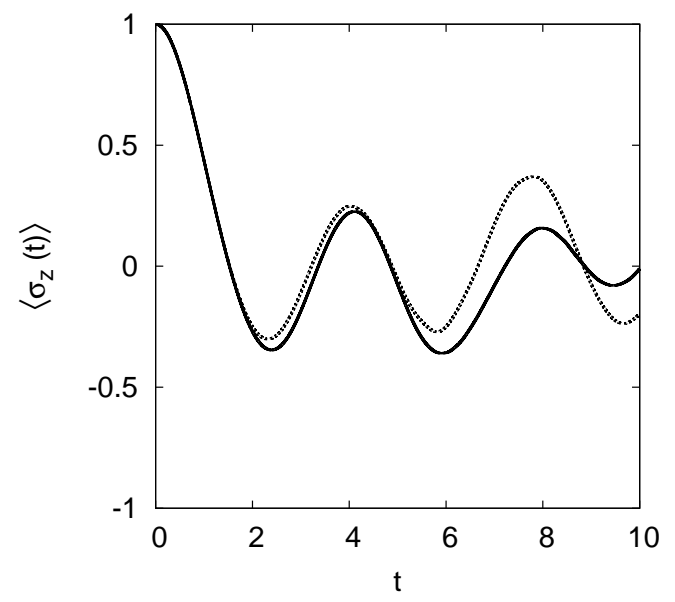

Figure 1: Time dependence of the population $\left\langle\sigma_{z}(t)\right\rangle$. The dotted line corresponds to the quantum-classical evolution while the solid-line corresponds to the quantum-corrected evolution. Frequency ratio $\Omega=0.6$, coupling constant $c=0.4$, initial average position $R_{0}=-1.6$, harmonic coefficient $b_{2}=-1$ and non-linearity parameter $b_{4}=0.5$.

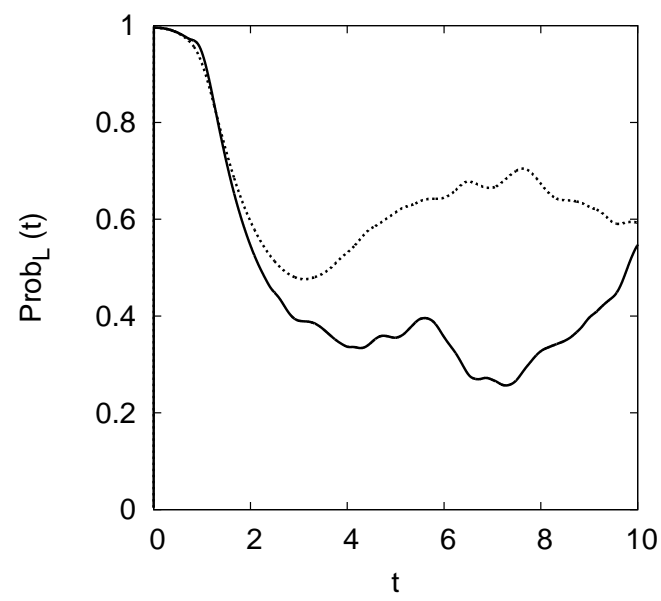

Figure 2: Time dependence of instantaneous oscillator left-well occupation probability $\operatorname{Prob}_{L}(t)$. The dotted line corresponds to the quantum-classical evolution while the solidline corresponds to the quantum-corrected evolution. Frequency ratio $\Omega=0.6$, coupling constant $c=0.4$, initial average position $R_{0}=-1.6$, harmonic coefficient $b_{2}=-1$ and non-linearity parameter $b_{4}=0.5$ 


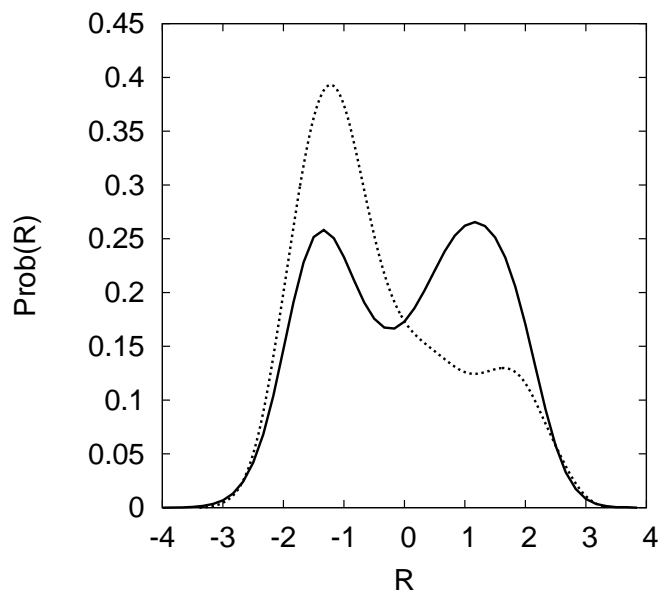

Figure 3: Oscillator position-probability $\operatorname{Prob}(R)$. The dotted line corresponds to the quantum-classical evolution while the solid-line corresponds to the quantum-corrected evolution. Frequency ratio $\Omega=0.6$, coupling constant $c=0.4$, initial average position $R_{0}=-1.6$, harmonic coefficient $b_{2}=-1$ and non-linearity parameter $b_{4}=0.5$

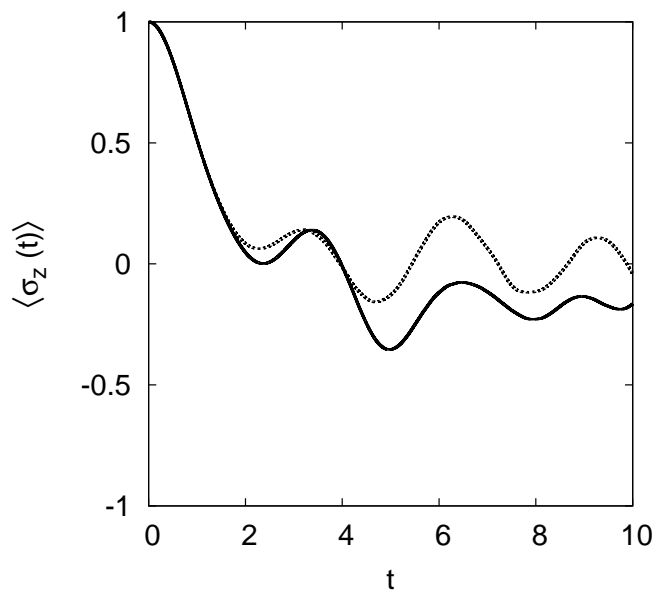

Figure 4: Time dependence of the population $\left\langle\sigma_{z}(t)\right\rangle$. The dotted line corresponds to the quantum-classical evolution while the solid-line corresponds to the quantum-corrected evolution. Frequency ratio $\Omega=0.6$, coupling constant $c=0.6$, initial average position $R_{0}=-1.6$, harmonic coefficient $b_{2}=-1$ and non-linearity parameter $b_{4}=0.5$. 


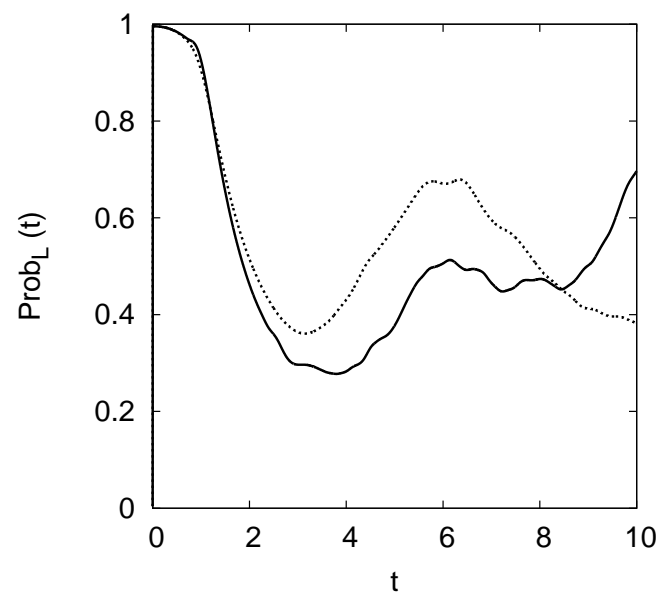

Figure 5: Time dependence of instantaneous oscillator left-well occupation probability $\operatorname{Prob}_{L}(t)$. The dotted line corresponds to the quantum-classical evolution while the solidline corresponds to the quantum-corrected evolution. Frequency ratio $\Omega=0.6$, coupling constant $c=0.6$, initial average position $R_{0}=-1.6$, harmonic coefficient $b_{2}=-1$ and non-linearity parameter $b_{4}=0.5$

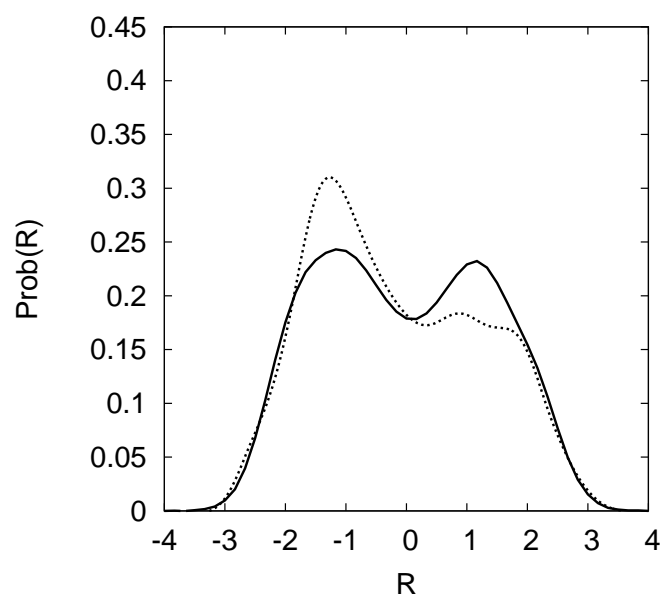

Figure 6: Oscillator position-probability $\operatorname{Prob}(R)$. The dotted line corresponds to the quantum-classical evolution while the solid-line corresponds to the quantum-corrected evolution. Frequency ratio $\Omega=0.6$, coupling constant $c=0.6$, initial average position $R_{0}=-1.6$, harmonic coefficient $b_{2}=-1$ and non-linearity parameter $b_{4}=0.5$ 


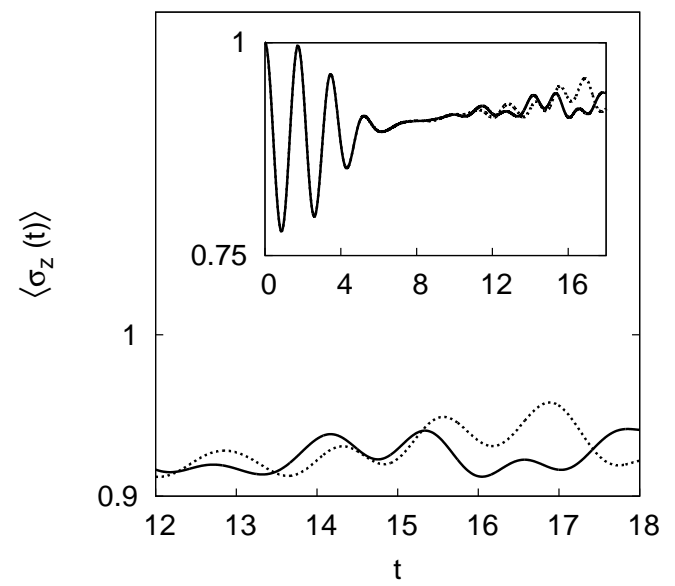

Figure 7: Time dependence of the population $\left\langle\sigma_{z}(t)\right\rangle$. The dotted line corresponds to the quantum-classical evolution while the solid-line corresponds to the quantum-corrected evolution. The inset displays the full time interval. Frequency ratio $\Omega=0.6$, coupling constant $c=0.1$, initial average position $R_{0}=-7.0$, harmonic coefficient $b_{2}=-0.01$ and non-linearity parameter $b_{4}=0.0004$. 